\title{
Improving the Data Transmission Throughput over the Home Electrical Wiring
}

\author{
Miguel Elias M. Campista, Luís Henrique M. K. Costa, and Otto Carlos M. B. Duarte \\ Grupo de Teleinformática e Automação \\ PEE-COPPE/DEL-POLI \\ Universidade Federal do Rio de Janeiro \\ C.P. 68504 - CEP 21945-970 \\ Rio de Janeiro - RJ - Brasil \\ E-mails: \{miguel,luish,otto\}@gta.ufrj.br
}

\begin{abstract}
Powerline communications (PLC) are receiving special attention since they use an already available and ubiquitous infrastructure. The main standard for PLC home networks is HomePlug. This work improves the throughput of HomePlug by modifying the medium access control sub-layer. The key idea is to define a fast collision avoidance mechanism where every station that wants to access the medium increments its contention window after sensing another ongoing transmission. The proposal reduces the number of collisions in the network improving the achievable throughput. We compared our mechanism to the original HomePlug standard through simulation and mathematical analysis. We verified that the improvement is independent from the packet size, the transmission rate and the number of nodes when the network is high loaded.
\end{abstract}

Index Terms-HomePlug, home networks, collision avoidance, medium access control.

\section{INTRODUCTION}

There is an increasing demand for connectivity in the home due to the need for Internet access and resource sharing at high rates. There are different contending home-network technologies. Among them, the "no-new-wires" solutions, such as HomePlug [1] and HomePNA [2], have the low cost and omnipresence as major advantages. Cabling may be decisive when choosing the home network to be installed, because it represents a large part of the final installation cost. HomePlug and HomePNA use, respectively, the existent electrical and telephone wiring infrastructure. A third lowcost home network solution is the wireless LAN, whose main standard is IEEE 802.11 [3].

The wireless technology has mobility as a singular feature. Nevertheless, wireless has problems related to attenuation and interference, which limit the bandwidth. Phone line home networks benefit from a dedicated medium between the central telephone station and the costumer. The phone line employees twisted pair wiring, which provides lower attenuation and lower interference than the electrical wires. Nevertheless, the number of phone outlets in a residence is usually smaller than the number of electrical outlets. Powerline Communication (PLC) home networks have ubiquity as a major advantage, because normally there are electrical outlets everywhere in a house. On the other hand, the electrical wiring was not designed for high-speed data transmissions, being a hostile medium for this end. According to Pavlidou et al., data transmission over the electrical medium tends to be worse than radio transmission in terms of attenuation and noise [4].

Possible solutions to cope with the electrical medium adversities are the subject of different works, which improve modulation, codification and signal processing techniques to allow higher data rates. Pavlidou et al. [4] and Biglieri [5] model the characteristics of the electrical medium for data transmission, noise and techniques employed in the communications through the electrical wiring. Other articles model the electrical medium based on the propagation characteristics and describes the echo model [6], [7]. The echo model calculates the medium transfer function taking into account the high number of reflections suffered by the electrical signal transmitted. These reflections are due to impedance mismatches.

This paper focuses on the HomePlug standard version 1.0 which defines the medium access method and the electrical physical layer specifications. Most works on HomePlug are limited to performance analysis of the protocol. Much work make comparative tests, experimental as well as theoretical, between the HomePlug and IEEE 802.11 medium access control (MAC) sub-layers [8], [9]. The protocols are compared in terms of maximum throughput achieved, connectivity, and throughput variation. The employment of HomePlug and IEEE 802.11 in the same home scenarios revealed a better performance of HomePlug, except in a few cases where IEEE 802.11 surpassed HomePlug. Lin et al. [8] present the standards, emphasizing HomePlug, and compare their average throughput and throughput variation while increasing the size of the links in a domestic environment. Lin et al. show that HomePlug achieves a higher average throughput and presents a higher stability due to lower throughput variation. Lee et al. [9] do a similar analysis in another home scenario reaching the same conclusions. The HomePlug throughput was analyzed mathematically by Jung et al. using a tri-dimensional discrete-time Markov chain [10]. Jung et al. considered that the network is saturated and deduced an expression for the HomePlug maximum throughput. Works aiming to improve the HomePlug performance are not yet found on the available literature.

The objective of this work is to improve the performance of 


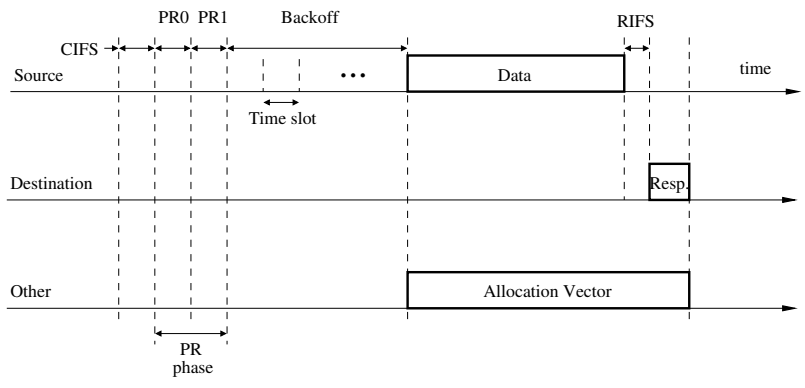

Fig. 1. Data frame transmission.

HomePlug by simple modifications to its collision avoidance mechanism. We propose and evaluate an improved mechanism for collision avoidance, which accelerates the protocol reaction against congestion. We demonstrate the efficiency of our scheme on the HomePlug standard through simulations and mathematical analysis.

This paper is organized as follows. Section II describes the HomePlug 1.0 standard and the corresponding MAC protocol. Section III presents the mechanism proposed and its mathematical evaluation which results in an expression for the collision probability. Section IV presents the results obtained through simulations and Section V concludes our work and presents future directions.

\section{THE HOMEPLUG STANDARD}

HomePlug 1.0 uses CSMA/CA (Carrier Sense Multiple Access with Collision Avoidance) to control the medium access. Collisions can not be detected because of the strong attenuation and noise [1]. In order to support quality of service, the standard defines four priority levels for the medium access, assigned according to the type of traffic. The priorities (Channel Access Priority - CAP) are associated to classes ranging from $\mathrm{CA} 0$ to $\mathrm{CA} 3$, where $\mathrm{CA} 3$ is the highest priority class.

A station that wants to transmit a data frame must first "listen" to the medium. To determine whether the medium is busy, the stations use physical carrier sense (PCS) and virtual carrier sense (VCS). Physical carrier sense alone cannot assure whether there is another ongoing transmission by only detecting preambles and priority assertions. Virtual carrier sense uses information from the frame "listened" to assert the transmission duration and to establish a virtual allocation vector. The stations will only transmit after the virtual allocation vector expiration.

To transmit a frame the medium must remain idle for at least a $35.84 \mu$ s time interval called CIFS (Contention Distributed InterFrame Space). After CIFS, the stations get into the Priority Resolution (PR) phase. Two time slots, Priority Resolution 0 (PR0) and Priority Resolution 1 (PR1), are used to allow attempts to access the medium only from higher priority flows during backoff, as depicted in Figure 1. The time intervals PR0 and PR1 also last for $35.84 \mu$ s each.

Before backoff, a station selects a random number uniformly distributed between zero and the contention window $(\mathrm{CW})$ size and instantiates a backoff counter (BC). The $\mathrm{BC}$ value chosen
TABLE I

HOMEPLUG VALUES FOR THE CONTENTION WINDOW (CW) SIZE AND DEFERRAL COUNTER (DC).

\begin{tabular}{|c||c|c|c|c|}
\cline { 2 - 5 } \multicolumn{1}{c|}{} & \multicolumn{2}{c|}{ CAP: CA3,CA2 } & \multicolumn{2}{c|}{ CAP: CA1,CA0 } \\
\hline BPC & CW & DC & CW & DC \\
\hline \hline 0 & 7 & 0 & 7 & 0 \\
\hline 1 & 15 & 1 & 15 & 1 \\
\hline 2 & 15 & 3 & 31 & 3 \\
\hline$\geq 2$ & 31 & 15 & 63 & 15 \\
\hline
\end{tabular}

times the time slot composes a random time interval used to initialize a backoff timer as shown in Figure 1. The time slot is a time interval defined by the HomePlug standard which lasts for $35.84 \mu \mathrm{s}$. The $\mathrm{CW}$ value depends on the number of times a backoff procedure was called during one frame transmission. The backoff procedure counter (BPC) stores the number of times the backoff procedure was called. During the contention periods, a station that wants to transmit a frame must wait for the medium to become idle for CIFS. After CIFS, the station transmits its priority signals at PR0 and PR1. If no station has higher priority, the station must start the backoff counter (BC) and wait for the backoff timer expiration to transmit. $\mathrm{BC}$ is decremented by one whenever the medium stays idle for a time slot. The values of the contention window size for the higher priority classes (CA3 and CA2) and for the lower classes (CA1 and CA0) are found in Table I.

The deferral counter (DC) is an additional mechanism conceived to avoid collisions by increasing the number of times the backoff procedure is performed for the frame being transmitted. Using DC, the backoff procedure may be called even if there was no collision. A station increases its backoff procedure counter (BPC) whenever a collision occurs, or when DC reaches zero. When DC is zero, every station contending for the medium must increment $\mathrm{CW}$ and postpone the transmission after sensing another station access. This mechanism reduces the collision probability. If $\mathrm{CW}$ and $\mathrm{DC}$ reach the maximum values defined by the standard (Table I), they are kept even if BPC is incremented.

The pseudo-code of the transmission algorithm using DC is shown in Figure 2. During a frame transmission, a station must wait for CIFS, and after the priority resolution period, wait for backoff. During backoff, a transmitter must listen to the medium, and if it remains idle, it transmits the frame. If another station transmits first, the station verifies its DC and decreases it by one if it is not zero. After decreasing DC, the station must pause its BC. The station resumes its BC and consequently its transmission if the medium gets idle for CIFS again and its priority allows. If DC is already zero, the station performs another backoff procedure and waits for the medium to get idle again for CIFS to restart a new backoff with a new $\mathrm{CW}$ value. After transmitting a data frame, the station waits for an acknowledgment (ACK). If the station does not receive an $\mathrm{ACK}$, it assumes a collision occurred and performs 


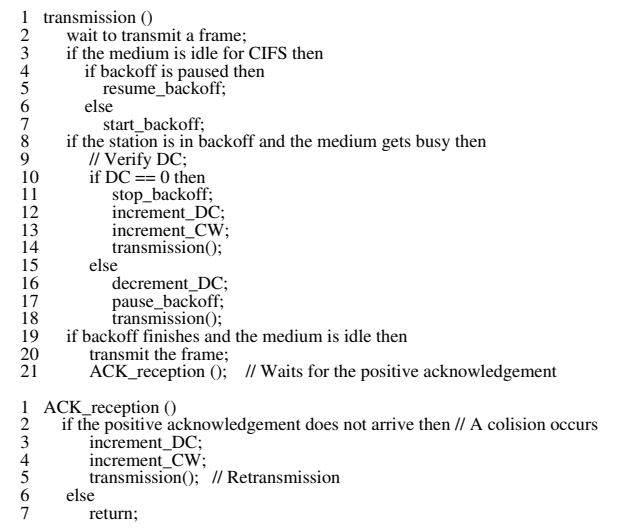

Fig. 2. The pseudo-code for transmission and reception of HomePlug using the deferral counter.

a backoff procedure. Then, the station waits for the medium to get idle again to retransmit. Otherwise, if an ACK is received, the station resets the minimum values for DC and CW.

\section{THE FAST COLLISION AVOIDANCE MECHANISM}

This paper proposes a novel mechanism based on the deferral counter (DC) to improve the collision avoidance in HomePlug. In the proposed fast collision avoidance mechanism, every station that wants to transmit must increment its contention window $(\mathrm{CW})$ size and choose a new backoff counter (BC) after sensing another ongoing transmission.

The backoff counter (BC) is a random number chosen in the interval $[0, C W]$ (Section II). A higher $\mathrm{CW}$ produces a higher range for $\mathrm{BC}$ values, preventing stations from selecting the same time slot to transmit. Intuitively, quickly increasing the $\mathrm{CW}$ decreases the collision probability. The Equation 1 evaluates, for the proposed mechanism, the probability that a station successfully transmits at any time slot during backoff. We suppose that each station chooses its BC independently.

In Equation 1, the indexes $i$ and $j$ are two transmitting nodes. Suppose that the set of nodes is denoted by $N$ set and that the number of nodes in the network is $n$. The time interval chosen for the transmission is $s$, and $W_{i}$ and $W_{j}$ are the current number of possible BC values for the transmission of nodes $i$ and $j$, respectively. The probability that a station $i$ to transmit in a time slot $s$ corresponds to the probability that a station $i$ chooses the time slot $s$ and the other stations $j$ select a time slot larger than $s$. The value of $W_{i}$ is equal to $C W_{i}+1$ of the station $i$ during the contention period.

$P_{t}=\frac{1}{W_{i}} \times \prod_{j \in N, j \neq i} \frac{W_{j}-s}{W_{j}}, i$ and $j \in N \mid N=\{1, \ldots, n\}$

If the values of $W_{j}$ increase for all stations $j$, the probability of one transmission in the time slot $s$ tends to $\frac{1}{W_{i}}$ when $W_{j} \rightarrow$ $\infty$. This result is equivalent to the probability that a station, transmitting alone, chooses any time slot to transmit among the $W_{i}$ possibilities. When $W_{j} \rightarrow \infty$, a station $j$ cannot choose a time slot equal to or lower than $s$ to transmit, thus $P_{t}$ tends to a transmission of a single station. If $W_{j} \rightarrow s \Rightarrow P_{t} \rightarrow 0$ because all stations collide. The number of stations also influences the transmission probability. The nodes $i$ and $j$ compete for the medium access as long as $W_{j}$ is similar to $W_{i}$. When the number of nodes tends to infinity, $P_{t} \rightarrow 0$ because $\frac{W_{j}-s}{W_{j}}<1$.

Generalizing Equation 1, the probability of a transmission occurrence in a contention period by any of the $n$ stations is given by Equation 2.

$$
P_{t}=\sum_{i=1}^{n} \frac{1}{W_{i}} \times \prod_{j \in N, j \neq i} \frac{W_{j}-s}{W_{j}} .
$$

The time slot $s$ must belong to the interval $\left[0, W_{\min }\right]$, where $W_{\min }=\min \left\{W_{0}, \ldots, W_{n}\right\}$. This restriction is necessary to guarantee that every multiplier in the product $\prod_{j \in N, j \neq i} \frac{W_{j}-s}{W_{j}}$ is greater than or equal to 0 . Otherwise, $P_{t}$ would be equal to 0 because the medium was already occupied by another transmission.

As a transmission can occur in any time slot $s$, we have:

$$
P_{t}=\sum_{s=0}^{W_{\min }} \sum_{i=1}^{n} \frac{1}{W_{i}} \times \prod_{j \in N, j \neq i} \frac{W_{j}-s}{W_{j}}
$$

In Equation 3, if $W_{j} \rightarrow \infty$, then $\sum_{i=1}^{n} \frac{1}{W_{i}} \times$ $\prod_{j \in N, j \neq i} \frac{W_{j}-s}{W_{j}}$ tends to one node transmission. In this case, all the sum components tend to zero but the one related to the transmission probability of the station $i$, which tends to $\frac{1}{W_{i}}$. Thus, $P_{t}=1$ because the transmission of this single node must happen in any time slot $s$.

Then, the collision probability $\left(P_{c}\right)$ is given by Equation 4 . Note that if $W_{j} \rightarrow \infty, P_{t} \rightarrow 1$ and $P_{c} \rightarrow 0$. Nevertheless, if the values of $W_{j} \rightarrow s$ or if $n \rightarrow \infty, P_{c} \rightarrow 1$. The proposed mechanism, accelerates the increase of $W_{j}$, therefore decreasing the collision probability. Equation 4 shows also that $P_{c}$ is independent of the packet size, supposing that every node is inside the same transmission range.

$$
P_{c}=1-\sum_{s=0}^{W_{\min }} \sum_{i=1}^{n} \frac{1}{W_{i}} \times \prod_{j \in N, j \neq i} \frac{W_{j}-s}{W_{j}} .
$$

The HomePlug standard applies a mechanism that reacts slower to probable collisions than the fast collision avoidance mechanism. The original HomePlug presents lower values for $C W$ than the proposed mechanism during transmissions. As a consequence, the collision probability (Equation 4) increases, justifying the employment of the fast collision avoidance mechanism. On the other hand, $C W$ can not increase indefinitely because it would increase the jitter as shown in Section IV.

\section{Simulations}

We developed simulation modules for the electrical medium and for the HomePlug 1.0 standard using the Network Simulator (ns-2), version 2.26 [11]. 


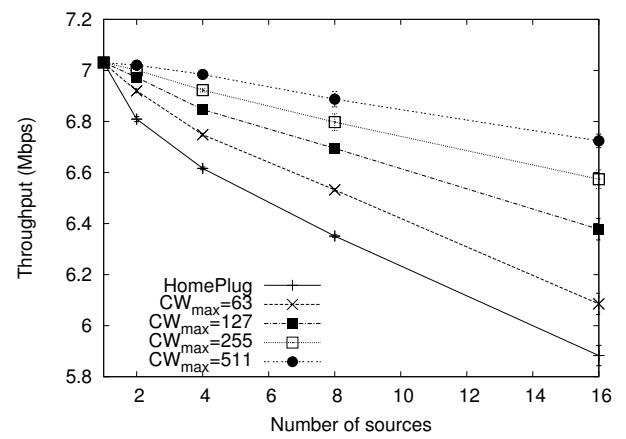

(a) 1500-byte packet throughput.

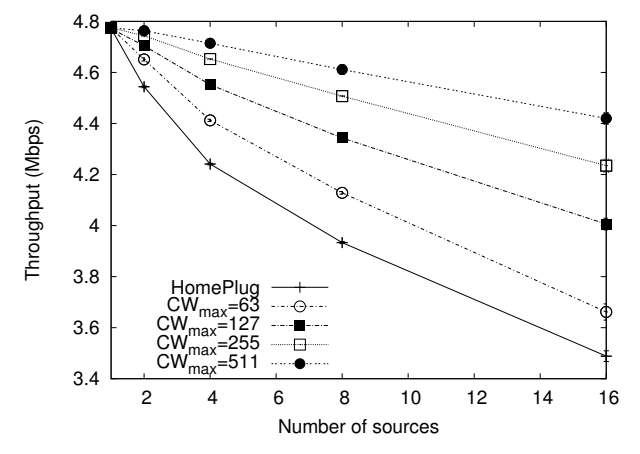

(b) 512-byte packet throughput.

Fig. 3. Throughput transmitting at $14 \mathrm{Mbps}$.

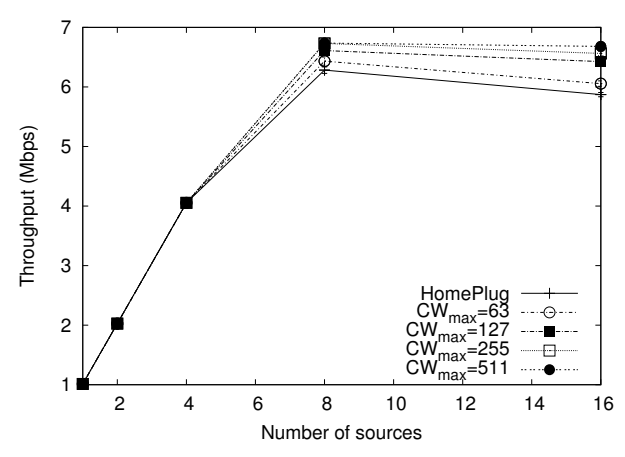

(a) 1500-byte packet throughput.

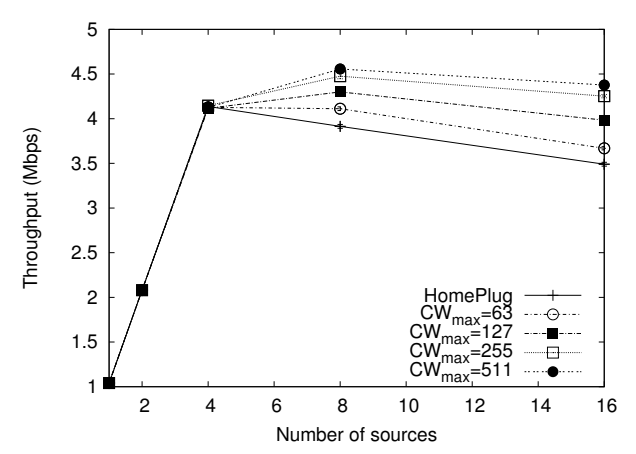

(b) 512-byte packet throughput.

Fig. 4. Throughput transmitting at $1 \mathrm{Mbps}$.

\section{A. Physical Layer}

We used the echo model described in [7] to model the physical layer, compute the attenuation and the transmission range. In the echo model, the signal received is the sum of the different components that may arrive out of phase and with different amplitudes. Due to the impedance mismatches, the signal transmitted arrives multiple times at the receiver, having followed multiple paths.

Also, we assume a bit error rate of $10^{-5}$ out of the decoders [12]. As a consequence, a receiver sends an NACK (Negative Acknowledgment) after detecting a frame with errors, causing the source to retransmit.

\section{B. Results}

The simulation scenario is composed of transmitting nodes that produce CBR over UDP traffic. Two packet sizes are used, 1500 and 512 bytes. All nodes are within the same transmission range, computed through the echo model mentioned in the previous section. The number of stations in the network varies from 1 to 16 . The offered load by each station may be 1 or $14 \mathrm{Mbps}$. The different packet sizes, number of stations, and individual offered loads are used to evaluate the efficiency of the proposed fast collision mechanism. Also, the behavior of the proposed mechanism is evaluated when modifying the maximum contention window $\left(C W_{\max }\right)$ size. Hence, $C W_{\max }$ ranges from 63 , which is the value of $C W_{\max }$ defined in HomePlug, to 511, according to the expression $C W_{\text {new }}=2 \times C W_{\text {current }}+1$, where $C W_{\text {current }}$ is the current $C W$, and $C W_{n e w}$ is the next value. In the following results, we computed confidence intervals of $95 \%$, which are represented in the graphs by vertical error bars.

Figures 3(a) and 3(b) evaluate the aggregated throughput of the network when every node transmits at $14 \mathrm{Mbps}$ using 1500 and 512-byte packets, respectively. The results show the advantage of the proposed mechanism when the network is under saturation. Higher throughput is achieved with larger packets. We also verify that the aggregated throughput decreases when the number of transmitting nodes increases, due to a higher number of collisions (Equation 4). Nevertheless, using the proposed mechanism, larger $C W_{\max }$ values reduce the throughput decrease. This result confirms Equation 3 which shows that when $\mathrm{CW}$ is increased the network throughput tends to the one-node transmission. The the throughput gains for the different $C W_{\max }$ curves are smaller for larger values of 


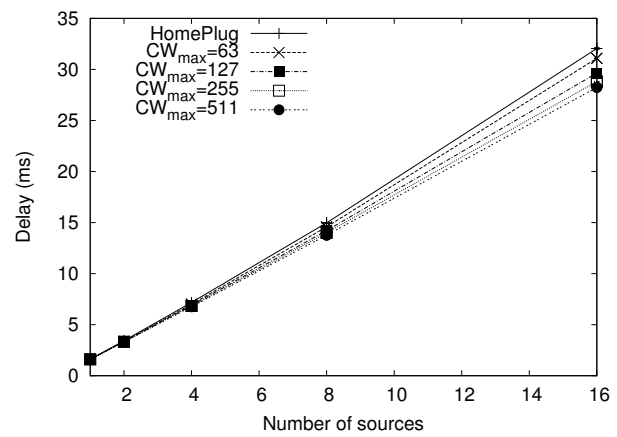

(a) 1500-byte packet delay.

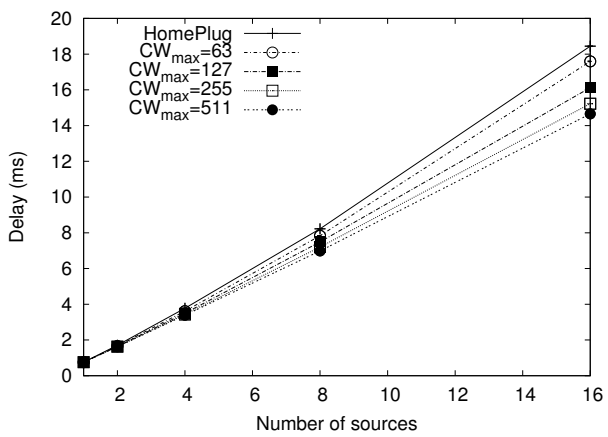

(b) 512-byte packet delay.

Fig. 5. Packet delay transmitting at 14 Mbps.

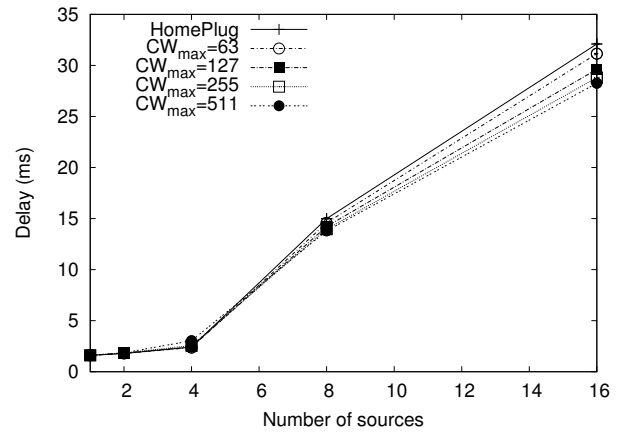

(a) 1500-byte packet delay.

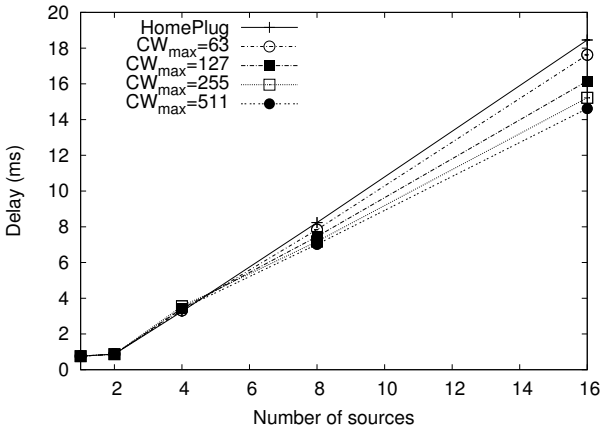

(b) 512-byte packet delay.

Fig. 6. Packet delay transmitting at $1 \mathrm{Mbps}$.

$C W_{\max }$. The increase of $C W_{\max }$ reduces the number of collisions, therefore the effect of high $C W_{\max }$ is not considerable if the number of collisions was already low. This behavior is independent from the packet size used. Nevertheless, the throughput achieved is higher for larger packets because the relative protocol overhead is smaller.

Figures 4(a) and 4(b) present the aggregated throughput when each node transmits at 1 Mbps using 1500 and 512byte packets, respectively. For the smaller numbers of nodes the network is not saturated. Thus, the aggregated throughput increases until the network saturates. When more than 4 nodes transmit 512-byte packets, the network saturates and the fast collision avoidance mechanism starts to work. For smaller packets the saturation is reached for a lower offered load. For 1500-byte packets, the throughput keeps increasing until 8 nodes when it saturates. It is observed that the network saturates first for smaller packets. This is due to a larger protocol overhead which results in a lower data transference. After saturation, the behavior of the curves becomes is similar to the curves for $14 \mathrm{Mbps}$ offered load. Before saturation, Figures 4(a) and 4(b) show that the behavior of HomePlug with and without the proposed mechanism is the same, because there is no collisions.

The delay is the average delay of all frames, measured from its reception from the MAC sub-layer of the source to the successful reception in the MAC sub-layer of the destination. If a collision or a channel error occurs during the packet transmission, the delay until its successful reception is taken into account. The propagation delay is negligible.

Figures 5(a) and 5(b) plot the average delay for 1500 and 512-byte packets, respectively, using sources that transmit at 14 Mbps. The average delay decreases for both packet sizes with the proposed mechanism. Increasing $\mathrm{CW}$ faster reduces the number of collisions. Even if the proposed mechanism causes a higher average medium access time, because $\mathrm{CW}$ is larger, the average delay is smaller for larger numbers of nodes. When the packet collides, the station has to retransmit. In average, the time needed to retransmit is larger than the time needed by the larger $\mathrm{CW}$ introduced by the proposed mechanism. Also note that for increasing $C W_{\max }$, the average delay decreases because the nodes that can not access the medium increase their $C W$ value faster reducing their access probability. Consequently, the number of stations that compete for the medium is temporarily smaller, these are the stations 


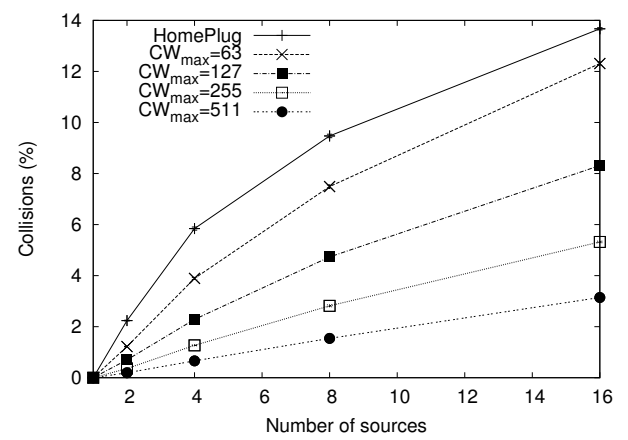

(a) 1500-byte packet collision probability.

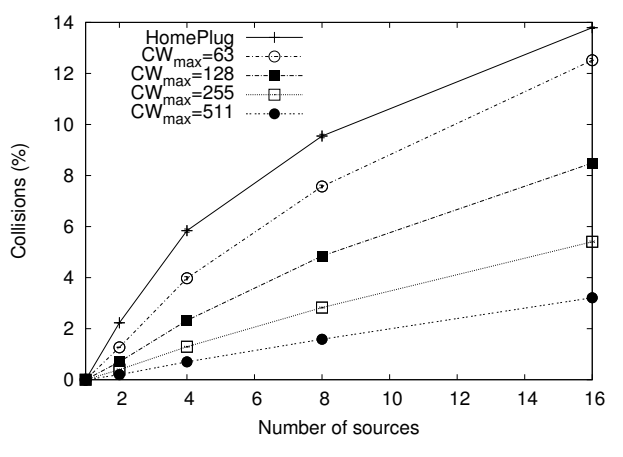

(b) 512-byte packet collision probability.

Fig. 7. Collision probability at 14 Mbps.

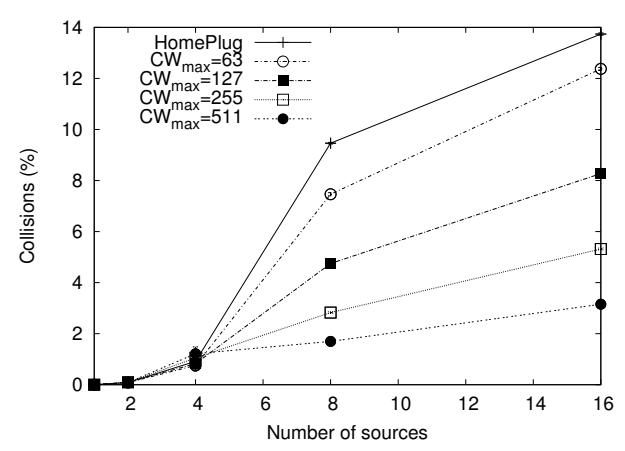

(a) 1500-byte packet collision probability.

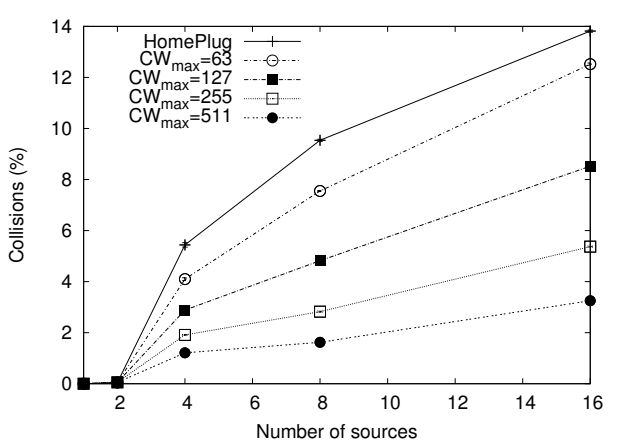

(b) 512-byte packet collision probability.

Fig. 8. Collision probability at 1 Mbps.

with smaller $\mathrm{CW}$ values. As the number of nodes competing is reduced, the average delay decreases. Although the number of retransmissions decreases, the jitter increases, as shown later. If the value of $C W_{\max }$ is indefinitely increased, the average delay tends to the average delay of one source, in accordance with Equations 3 and 4 . Note also that the difference between the curves for different $C W_{\max }$ values decreases as $C W_{\max }$ increases.

Figures 6(a) and 6(b) show the average delay when each node transmits at $1 \mathrm{Mbps}$. It is observed that the average delay increases when the network saturates. For 512-byte packet transmission the saturation occurs first, as seen in Figures 4(b) and 3(b). The average delay starts to grow for 2 sources transmitting 512-byte packets, and for 4 sources with 1500-byte packets. Again, for smaller packets the protocol overhead is higher then the saturation is reached with lower network load. After saturation, the behavior is analogous to the $14 \mathrm{Mbps}$ offered charge scenario. Before saturation, both HomePlug and proposed mechanism present the similar average delays.

A collision occurs whenever more than one station transmits in the same time slot. The collision probability is defined as the number of collisions divided by the total number of transmissions. Figures 7(a) and 7(b) show that transmitting at $14 \mathrm{Mbps}$, independently of the packet size, the collision probability is the same. Although the number of collisions is larger for small packets due to the higher number of medium accesses, the number of transmissions is also higher. Thus, the probability of a collision is the same when compared to larger packets. With increasing $\mathrm{CW}$ values, the collision probability decreases because the probability that at least two nodes choose the same slot to transmit is reduced. Very large values of $\mathrm{CW}$ results in a collision probability close to zero.

Figures 8(a) and 8(b) show the collision probability for an offered load of $1 \mathrm{Mbps}$ per node. After saturation, the collision probability is similar for both packet sizes, which confirms that the probability of collisions is independent of the packet size. Also, note that once saturation is reached, the collision probability is also independent of the sending rate of the sources. Again, the saturation arrives first with smaller packets.

We also analyzed the effects of the $\mathrm{CW}$ increase and the number of retransmissions over the jitter. Retransmissions add to the jitter, because larger times are needed to successfully 


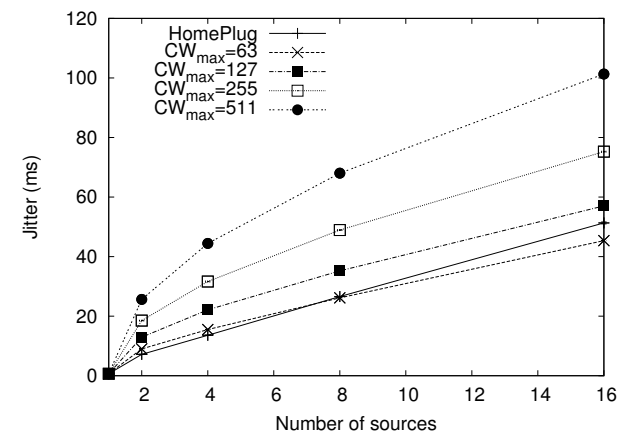

(a) 1500-byte packets.

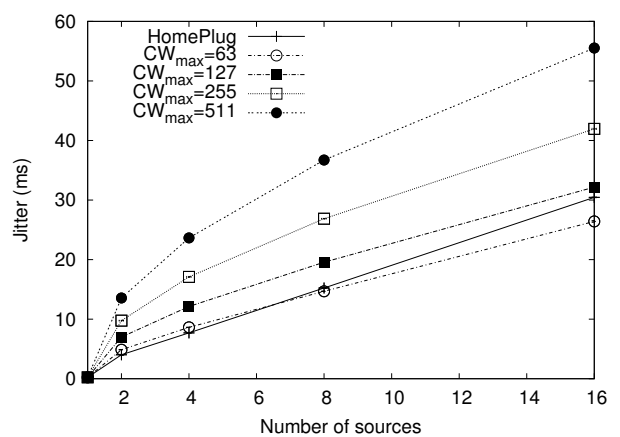

(b) 512-byte packets.

Fig. 9. Jitter at 14 Mbps.

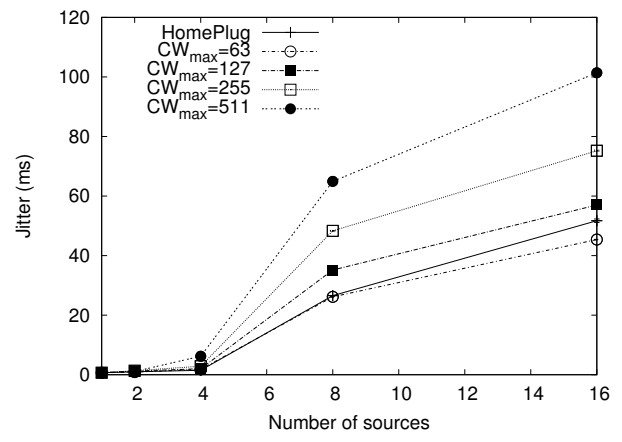

(a) 1500-byte packets.

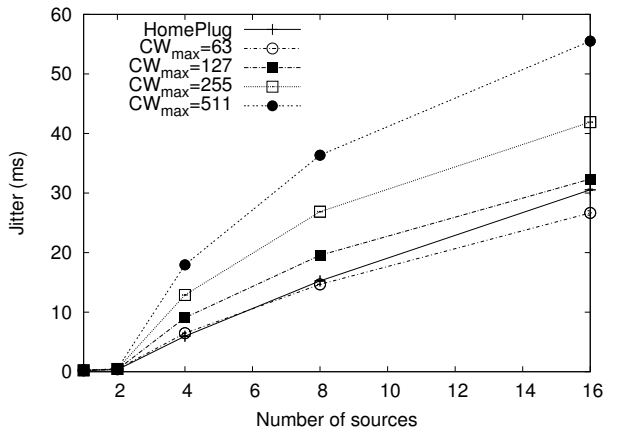

(b) 512-byte packets.

Fig. 10. Jitter at 1 Mbps.

transmit. Nevertheless, increasing $\mathrm{CW}$ faster also adds to the jitter because the $\mathrm{CW}$ enlargement reduces the probability that a station with a higher $\mathrm{CW}$ value chooses a backoff counter (BC) small enough to access the medium. Thus, after successive unsuccessful attempts, the delay will be high. If a transmission succeeds, the probability of another transmission by the same station increases, since its $\mathrm{CW}$ will be minimum $\left(C W=C W_{\min }\right)$ and consequently the delay will be low. Thus, the proposed mechanism also contributes to the jitter. The jitter increase is larger for higher $C W_{\max }$ values because the nodes that do not access the medium tend to additionally postpone their transmissions.

Figures 9(a) and 9(b) show the jitter when the stations transmit at $14 \mathrm{Mbps}$. The proposed mechanism with $C W_{\max }=63$ reduce the jitter compared to the standard HomePlug for a higher load, independently of the packet size. Therefore, the effect in the jitter of the higher number of retransmissions overcomes the effect of the $\mathrm{CW}$ fast increase. Nevertheless, when $C W_{\max }>63$, the modified HomePlug has a higher jitter. In this case, the effect of the fast increase of $\mathrm{CW}$ exceeds the effect of retransmissions. Thus, there is a tradeoff between the jitter and the collision probability, or, ultimately, a tradeoff between the jitter and the throughput.

In Figures 10(a) and 10(b), the sources transmit at $1 \mathrm{Mbps}$. The jitter behavior is the same of Figures 9(a) and 9(b) after saturation.

Figures 9(a) to 10(b) show that the jitter of the standard HomePlug is the one which increases faster. With higher numbers of nodes, the number of collisions increases faster for the standard HomePlug than for the proposed mechanism. This is because HomePlug with the proposed modification reaches higher $\mathrm{CW}$ values faster than the standard HomePlug. Therefore, the fast collision avoidance mechanism achieves a collision probability equivalent to the original HomePlug only for a higher number of sources. This number of sources increases with the value of $C W_{\max }$. Depending on the number of sources and on the network load, using $C W_{\max }>63$ may be a better solution. Figures 9 (b) and 10(b) show that for high load the jitter between HomePlug and the proposed mechanism is approximately the same for $C W_{\max }=127$. This happens when the number of sources is 16 , which represents the worst scenario analyzed in terms of saturation. Figures 9(a), 9(b), 10(a), and 10(b) show that an increasing number of collisions influences more the jitter than the faster 
$\mathrm{CW}$ increase. This is due to a faster increase in jitter of the original HomePlug when $C W_{\max }=63$ compared to the proposed mechanism with the same $C W_{\max }$.

We observed that using $C W_{\max }=63$ the performance is better under all the aspects analyzed, except when the network load is high enough to make $C W_{\max }>63$ a better solution as in Figures 9(a), and 9(b) for 16 sources. Besides, using HomePlug with the proposed mechanism with $C W_{\max }$ set to 63 is easier to implement.

The performance of the network can be improved if $C W_{\max }$ is adjusted according to the to the network load and the number of sources. The $C W_{\max }$ value could be previously configured in the network card driver or could be chosen adaptively. To appropriately change the card configuration before transmissions, a previous knowledge of the number of nodes and of the load of the network is needed. The other approach the driver could be reconfigured according to the network conditions. In this case, the driver must be able of dynamically measuring the current load. The complexity of choosing the $C W_{\max }$ value is related to the knowledge of the parameters needed to adapt the node to the current network load.

\section{Conclusions}

This work proposed a collision avoidance mechanism to improve the throughput of the HomePlug MAC protocol. In the proposed mechanism, differently from HomePlug, a station that wants to transmit always increments its contention window $(\mathrm{CW})$ when it listens that the medium was occupied by another station. The value of $\mathrm{CW}$ is incremented until a maximum value $\left(C W_{\max }\right)$ is reached, independently from the transmission success. The proposed mechanism can be easily implemented since it requires a small modification to the MAC sub-layer.

A mathematical analysis derived an expression for the collision probability. The derived expression shows that for a large number of nodes the collision probability increases. It was also shown that increasing the $C W_{\max }$ values the network throughput tends to the throughput achieved by a single node transmitting. These results encouraged us to propose a novel mechanism able to increase the contention window faster and to test higher $C W_{\max }$ values.

The proposed mechanism was incorporated into the HomePlug module, which we implemented for the ns-2 simulator. The simulations verified that the fast collision avoidance mechanism improves the HomePlug throughput for different packet sizes, number of nodes in the network, and sending rates. The relative throughput gains are larger for high network load. We also analyzed the effect of using larger values for the maximum contention window $\left(C W_{\max }\right)$. We varied $C W_{\max }$ from one to eight times the original $C W_{\max }$ defined in the HomePlug standard. We verified that increasing $C W_{\max }$, the throughput of the network tends to that of only one node transmitting. Larger $C W_{\max }$ values increased the network throughput and reduced the average delay and number of collisions. The tradeoff is that the $C W_{\max }$ increase also increases the jitter of the network. Also, it was demonstrated that the $C W_{\max }$ value that shows the best performance depends on the network load.

We concluded that the use of the proposed mechanism with $C W_{\max }=63$, as defined in the standard, produces performance gains taking all the aspects analyzed into account. A value of $C W_{\max }>63$ would be advantageous only for very high network loads when the jitter achieved by the original HomePlug is the same achieved by the proposed mechanism with higher $C W_{\max }$. Our future work will investigate the implementation of a dynamic mechanism which is able to adapt the $C W_{\max }$ to the network load.

\section{ACKNOWLEDGMENTS}

CNPq, CAPES, FINEP, FAPERJ, FUNTTEL, and RNP have supported this work.

\section{REFERENCES}

[1] M. K. Lee, R. E. Newman, H. A. Latchman, S. Katar, and L. Yonge, "Homeplug 1.0 powerline communications LANs - protocol description and performance results," International Journal of Communication Systems, vol. 16, no. 5, pp. 447-473, jun 2003.

[2] ITU-T, "G.989.1: Phoneline networking transceivers - foundation," feb 2001, recommendation.

[3] IEEE, "Wireless LAN medium access control (MAC) and physical layer (PHY) specifications," 1999, IEEE Standard 802.11.

[4] N. Pavlidou, A. J. H. Vinck, J. Yazdani, and B. Honary, "Power line communications: State of the art and future trends," IEEE Communications Magazine, vol. 41, no. 4, pp. 34-40, apr 2003.

[5] E. Biglieri, "Coding and modulation for a horrible channel," IEEE Communications Magazine, vol. 41, no. 5, pp. 92-98, may 2003.

[6] P. Langfeld, "The capacity of typical powerline reference channels and strategies for system design," in International Symposium on PowerLine Communications and its Applications (ISPLC), Malmö, Suécia, apr 2001, pp. 271-278.

[7] K. Dostert, "Power lines as high speed data transmission channelsmodelling the physical limits," in IEEE 5th International Symposium on Spread Spectrum Techniques and Applications, vol. 2, sep 1998, pp. 585-589.

[8] Y.-J. Lin, H. A. Latchman, and R. E. Newman, "A comparative performance study of wireless and power line networks," IEEE Communications Magazine, vol. 41, no. 4, pp. 54-63, apr 2003.

[9] M. K. Lee, H. A. Latchman, R. E. Newman, S. Katar, and L. Yonge, "Field performance comparison of IEEE 802.11b and Homeplug 1.0," in IEEE Conference on Local Computer Networks (LCN'O2), nov 2002, pp. 598-599.

[10] M.-H. Jung, M. Y. Chung, and T.-J. Lee, "MAC Throughput Analysis of HomePlug 1.0," IEEE Communications Letters, vol. 9, no. 2, pp. 184-186, feb 2005.

[11] K. Fall and K. Varadhan, The ns Manual, UC Berkeley, LBL, USC/ISI, and Xerox PARC, apr 2002, available at http://www.isi.edu/nsnam/ns/nsdocumentation.html.

[12] J. Foerster and J. Liebetreu, "FEC Performance of Concatenated ReedSolomon and Convolutional Coding with Interleaving," IEEE, Tech. Rep., June 2000, http://ieee802.org/16/phy/contrib/802161pc-00_33.pdf. 